about the disease and encourage them to be vaccinated. University representatives have said that because many universities don't have the money to pay for vaccination, it is unlikely they will mandate such vaccinations prior to admission.

The American Academy of Pediatrics and the U.S. Public Health Service's Advisory Committee on Immunization Practices currently recommend hepatitis B immunization for all infants and older children, adolescents, and adults at increased risk of infection. Both groups also encourage routine immunization of all adolescents.

\section{Latex Allergies Reported by OR Nurses}

Latex glove sensitivities were reported by 369 (21\%) of the 1,738 operating room (OR) nurses recently surveyed at an Association of Operating Room Nurses (AORN) annual convention. The findings of this questionnaire survey were reported by Dr. Stephanie Zaza, medical epidemiologist at CDC, at this year's Epidemic Intelligence Service (EIS) conference in Atlanta, Georgia. Those nurses reporting reactions to latex were more likely than those without such reactions to have a history of other allergies, asthma, and chronic illnesses. The reported symptoms included itching $(86 \%)$, rash $(54 \%)$, dermatitis (51\%), hives (13\%), facial swelling (4\%), and wheezing (1\%). Symptoms were reported most frequently after contact with latex gloves, sterile or nonsterile, powdered or unpowdered. These findings add to the mounting evidence that potentially severe latex glove allergies appear to be increasing.

Although many of the reactions appeared to be related to individual risk factors for allergies, additional studies are needed to determine whether specific types of gloves could be particularly allergenic.

\section{New Technique to Detect Small Amounts of HIV in Blood Cells}

It is now possible to detect HIV DNA or RNA in a small number of blood cells using a highly sensitive new assay technique developed by Dr. Bruce Patterson and colleagues at Northwestern University Medical School in Chicago. The technique involves the use of polymerase chain reaction (PCR) to amplify HIV nucleic acids within cells, tagging them with a fluorescent probe and analyze them using flow cytometry, This new assay allows researchers to quantify the number of HIV-infected cells in peripheral blood and to isolate them for further characterization. This new assay is able to identify HIV genetic material in as few as one in 10,000 blood cells compared with previous assays that could detect HIV in only one in ten blood cells.

FROM: Patterson BK et al. Science 1993;260:976979.

\section{PCR Test Kit for Detection of Chlamydia Awaits FDA Clearance}

The use of polymerase chain reaction (PCR), a new tool in molecular biology, may soon be adding the detection of chlamydia to its list of applications. Roche Molecular Systems, Inc., a division of Hoffmann-La Roche, has submitted a PCR test kit for detection of chlamydia to the FDA and is awaiting clearance. Hoffmann-Ia Roche recently acquired full rights to PCR technology from Cetus Corp. and now owns all technical, patent, and manufacturing rights to all known and future uses for PCR. PCR testing is currently available for HIV, HTLV-I and HTLV-II, Borrelia burgdorferi (Lyme disease), mycobacteria, and paternity testing through Roche Biomedical Laboratories. PCR research technology also has yielded promising results in amplifying the genetic sequences responsible for cystic fibrosis and Duchenne's muscular dystrophy.

\section{EcoliO157:H7 Transmission Reported in Minnesota Daycare Centers}

Although only two reports of Escherichia coli O157:H7 outbreaks in child daycare centers had been published previously, the Minnesota Department of Health identified 68 cases of E coli $0157: H 7$, including 29 cases at nine daycare centers with evidence of person-to-person transmission in all nine daycare centers. Cases of $\mathrm{E}$ coli $\mathrm{O} 157: \mathrm{H} 7$ infection were identified by interviewing parents of infected children under 5 years of age from July 1988 through December 1989. If the child attended a daycare after onset, stool cultures were obtained from other children in attendance and their parents were interviewed. If there was presumptive evidence of ongoing $E$ coli 0157:H7 transmission in a facility, all preschool children were excluded from attending daycare centers until two consecutive stool cultures were negative. There was no evidence of further transmission at centers where children were excluded temporarily until two consecutive stool cultures were negative.

The authors concluded that the number of unrecognized daycare outbreaks in the United States may 